\title{
Tendencias y perspectivas de desarrollo rural en la provincia de Segovia: oportunidades y límites
}

\author{
Luis Carlos MARTínez FERNÁNDEZ \\ Departamento de Geografía \\ Universidad de Valladolid \\ luiscar@fyl.uva.es
}

Recibido: 24 de enero de 2014

Enviado a evaluar: 26 de enero de 2014

Aceptado: 27 de abril de 2015

\section{RESUMEN}

La provincia de Segovia está inmersa en un proceso de transformación de sus estructuras productivas que tiene que ver, como primera manifestación, con la actividad agraria y el desarrollo rural. La calidad de las producciones alimentarias constituye una de las apuestas más seguras con la que enfocar el futuro del predominante mundo rural segoviano. En él, la dinamización económica y el progreso de la diversificación de funciones tienen que ver, más que con cualquier otra consideración, con una creciente terciarización en base a los servicios de atención a la menguante población. Son las limitaciones y las oportunidades que se ciernen sobre el desarrollo rural provincial. Tendencias apuntadas y perspectivas de futuro que encuentran en el entramado agroindustrial y en los productos agrarios de calidad su principal vector.

Palabras clave: Espacio rural, calidad agroalimentaria, pluriactividad, desarrollo rural, Segovia.

\section{Tendences and prospects for rural development in the province of Segovia: opportunities and limitations}

\begin{abstract}
The province of Segovia (Castilla y León, Spain) is immersed in a process of transformation of their production structures that has to do, as the first manifestation, with agricultural activity and rural development. The quality of the agro-food production is one of the safest bets with which to approach the future of predominant rural environment of Segovia. In it, the economic dynamization and progress of the diversification of functions have to do more, than any other consideration, with an increasing tertiarisation based on attention services to the decreasing population. They are the limitations and opportunities posed to the provincial rural development. Trends pointed and future perspectives found in the agroindustrial framework and quality agricultural products their main vector.
\end{abstract}

Key words: Rural space, agro-food quality, pluriactivity, rural development, Segovia (Spain). 


\section{Tendences et perspectives de développement rural dans la province de Segovia: opportunités et limitations}

\section{RESUMÈ}

Les structures productives de la province de Segovia (Castilla y León, Espagne) sont aujourd'hui en processus de transformation; ce qui est associé, premièrement, aux activités agraires et au développement rural. La qualité des productions alimentaires constitue un solide pari pour regarder l'avenir du monde rural de Segovia. La dynamisation économique et la diversification de fonctionnes y ont un lien avec une croissante tertiarisation basée sur les services; mais la population diminue. Ceux-ci sont les limitations et les opportunités du développement rural de la province. Des tendances observées et des perspectives dont le principal vecteur sont les industries agraires et leurs produits d'une qualité prouvée.

Mots-clés: Espace rural, qualité agroalimentaire, pluriactivité, développement rural, Segovia (Espagne).

\section{INTRODUCCIÓN}

En el camino inexorable hacia el "post" o "neo" productivismo (Armesto, 2005; Ilbery y Bowler, 1998), más que la capacidad productiva, que también, siendo constado este hecho como evidente para las producciones y las explotaciones más rentables (mientras las menos quedan a merced de la "asistencia" vía pagos de la $\left.\mathrm{PAC}^{1}\right)$, la calidad de las producciones alimentarias constituye la apuesta más segura

${ }^{1}$ En realidad, las ayudas directas de la Política Agrícola Común en apoyo compensatorio al declive de las rentas agrarias en Europa (las correspondientes al denominado "primer pilar", frente a las del "segundo pilar" o medidas de desarrollo rural) llegan a todos los agricultores y ganaderos segovianos titulares de explotación, con independencia del grado de "profesionalización" y si la actividad es principal o secundaria (es más, para el nuevo periodo de programación 2014-2020 se contempla que un agricultor "activo" es quien obtenga, al menos, un $20 \%$ de los ingresos de la actividad y el resto de subvenciones). Es así como este auténtico y valioso "seguro" agrario cubre por igual a los agricultores y ganaderos profesionales que a los "extractores de rentas del campo" (en términos de Fernando Molinero). Por consiguiente, no dejan de cobrar importancia tanto los jubilados de la actividad, que ven compensadas, de esta forma, sus contenidas prestaciones contributivas, como los ocupados en otros sectores, que tienen en la explotación agraria -vía subvención- su fuente de complemento de rentas. Con todo, la disponibilidad de pagos compensatorios también ha sido determinante para el mantenimiento y consolidación de una agricultura con carácter empresarial y competitivo (Molinero et al., 2012, 435). De los 8.780 perceptores de las ayudas directas de la PAC en 2009 (8.656 en 2013), el 53,27\% lo hacían (con ese carácter de complemento en las explotaciones más pequeñas) en cantidades inferiores a 5.000 euros anuales, el 40,15\% percibía entre 5.000 y 30.000 euros y, únicamente, un 2,36\%, el de los "profesionales" más dinámicos, superaban los 50.000 euros. Unas ayudas de la PAC que aparecen vinculadas, fundamentalmente, al sector ganadero ("Derechos de Pago Único", primando la extensificación y a las vacas nodrizas), a la rotación de cultivos, las leguminosas de calidad y al azúcar: "La Junta autoriza el pago de 35 millones de euros en ayudas de la PAC para toda la provincia”. El Adelantado de Segovia, 21 de diciembre de 2013. 
con la que enfocar el futuro de la actividad agraria segoviana (Martínez, 2014). Se hace así evidente, pues, que frente a la regulación, los cupos y las cortapisas impuestas a las producciones masivas por la PAC -las contrapartidas de la subvención-, y a partir de una creciente demanda capaz, como consecuencia de la mejora económica de determinados segmentos de la sociedad, la calidad se convierte en una opción clave de desarrollo (agrario) rural (Tolón y Lastra, 2009).

En un espacio provincial inmerso en un intenso proceso de transformación de su entramado productivo: un verdadero espacio en transformación, como ha venido a ser definido por los estudiosos preocupados por el desarrollo provincial (García, 2010), la realidad del desarrollo rural pasa, como primer aspecto a destacar, por el notable dinamismo atribuido a la actividad agroalimentaria, merced al relevante grado de modenización que han alcanzado sus estructuras más representativas, y que se incorpora ya, como una dimensión más, a la dinamización económica y al progreso de la diversificación de funciones del predominante mundo rural segoviano.

\section{LA CALIDAD AGROALIMENTARIA COMO APUESTA DE FUTURO}

El interés cultural, la conservación de variedades vegetales o animales y el patrimonio técnico de elaboración son los valores añadidos, frecuentemente esgrimidos, que emanan de este tipo más restringido de producción ${ }^{2}$.

A caballo entre el control de la excelencia y la promoción comercial, destacando las vinculaciones con el territorio, las variedades genéticas o incidiendo en los procesos de transformación, son varias las figuras, que integradas en el marco normativo de la Unión Europea, fijan los estándares y configuran las "marcas" más vendibles como producciones agroalimentarias de calidad (Arfini, 2006; Galdos, 2004; Muchnik, 2006).

Las Denominaciones de Origen Protegidas (DOP) constituyen el nivel más alto de control, aprecio, garantía y entidad económica. Le siguen, en importancia cualitativa, las Indicaciones Geográficas Protegidas (IGP), de creación más reciente, que acogiendo productos igualmente afamados y vinculados en su elaboración a un ámbito espacial concreto, no son tan exigentes ni precisan de tanta concreción en lo que respecta a las variedades o al origen de las materias primas animales o vegetales. Con menos entidad y bajo condiciones más laxas son contempladas otras figuras, caso de los Vinos de Calidad Producidos en una Región Determinada (VCPRD), los Vinos de la Tierra o, en sentido más amplio, las Marcas de Garantía, que destacan

\footnotetext{
${ }^{2}$ Es el hilo argumental que se sigue en el inventario de productos agroalimentarios de calidad más completo que se ha hecho para Castilla y León (Molinero et al., 2001).
} 
cualquier singularidad ${ }^{3}$. Unas y otras figuras de protección y de comercialización han venido teniendo una acogida extraordinaria en España y, particularmente, en Castilla y León, al tiempo que conocen una expansión acorde con el peso que están llamadas a tener en la dinamización económica y la diversificación de funciones dentro del mundo rural de esta Comunidad (Molinero, 2012). Y en ello, Segovia no ha de ser -ni lo es- una excepción. Como lo ponen de manifiesto las respuestas dadas a las preguntas que sobre este particular se formulan en la Encuesta a los Municipios de Segovia realizada en marzo de 2014 por el Departamento de Geografía de la Universidad de Valladolid, y en la que se constata que la presencia de la actividad agroganadera de calidad es percibida en un $60,36 \%$ de los municipios que han contestado a esta cuestión (Tabla 1); haciéndose constatar, además, como en la mayoría de los casos $(93,10 \%)$ la actividad es generadora de algún tipo de expectativa laboral, con efectos que trascienden, también, al conjunto de sectores de la economía local.

Tabla 1. Percepción sobre la importancia de la actividad agroganadera de calidad en la provincia de Segovia.

\begin{tabular}{|c|c|c|c|c|c|c|}
\hline \multirow[t]{2}{*}{ Pregunta } & \multicolumn{3}{|c|}{$\begin{array}{l}\% \text { sobre los } 121 \text { municipios } \\
\text { encuestados }\end{array}$} & \multicolumn{3}{|c|}{$\begin{array}{l}\% \text { sobre el total de respuestas a la } \\
\text { pregunta }\end{array}$} \\
\hline & SI & NO & $\%$ & SI & NO & $\%$ \\
\hline $\begin{array}{l}\text { ¿Hay en su municipio o en los de su entorno } \\
\text { algún producto alimentario (agrícola o } \\
\text { ganadero) de singular calidad? }\end{array}$ & $55,37 \%$ & $36,36 \%$ & $91,73 \%$ & $60,36 \%$ & $39,64 \%$ & $100 \%$ \\
\hline $\begin{array}{l}\text { ¿Ha creado empleo o podría crearlo en un } \\
\text { futuro cercano? }\end{array}$ & $44,63 \%$ & $3,31 \%$ & $47,93 \%$ & $93,10 \%$ & $6,90 \%$ & $100 \%$ \\
\hline \multirow{2}{*}{ Pregunta } & \multicolumn{6}{|c|}{$\%$ sobre el total de respuestas afirmativas a la pregunta } \\
\hline & \multicolumn{2}{|c|}{ Actividad agraria } & \multicolumn{2}{|c|}{ Industria } & \multicolumn{2}{|c|}{ Servicios } \\
\hline ¿Ha creado empleo en...? & \multicolumn{2}{|c|}{$61,06 \%$} & \multicolumn{2}{|c|}{$29,20 \%$} & \multicolumn{2}{|c|}{$9,74 \%$} \\
\hline ¿Podría crear empleo en...? & \multicolumn{2}{|c|}{$41,12 \%$} & \multicolumn{2}{|c|}{$41,12 \%$} & \multicolumn{2}{|c|}{$17,76 \%$} \\
\hline
\end{tabular}

Fuente: Departamento de Geografía de la Universidad de Valladolid: Encuesta a los Municipios de Segovia, 2014.

El Instituto Tecnológico Agrario de Castilla y León (ITACyL) es el órgano encargado de gestionar la política de inspección, control y apoyo a los Consejos Reguladores de productos amparados por las figuras de calidad agroalimentarias

${ }^{3}$ Como ocurre, por ejemplo, con la Marca de Garantía Setas de Castilla y León o la Marca de Garantía del Queso de la Región del Duero, a la que se incorpora la producción lechera segoviana (de vaca, oveja y cabra) que tenga por fin la elaboración del queso castellano a la manera tradicional (puro de oveja; queso de vaca, cabra y oveja; y queso de vaca y oveja). De carácter más específicamente segoviano, apuntar la reciente aprobación de la Marca de Garantía Judión de La Granja. 
dentro del territorio regional. Su actividad se basa en dos estrategias fundamentales: el fomento de la buena utilización de los distintivos de origen y calidad oficiales de los alimentos, por él registrados, y la promoción de estos distintivos a fin de que sean reconocidos y considerados por todos los consumidores. En este sentido, ante la gran dispersión de las figuras de calidad impuesta por la gran extensión de Castilla y León y dada la calidad singularizada de sus producciones, el Instituto ha diseñado un Distintivo de calidad alimentaria que, aparte de velar por el interés general de asegurar al público la procedencia y calidad garantizada de estos productos de origen regional, facilite una campaña de comercialización y promoción conjuntas de la producción agraria de las nueve provincias de la Comunidad. Esta distinción es la imagen de marca conocida como Tierra de Sabor ${ }^{4}$. Y a ella se adscriben las figuras agroalimentarias de calidad de las que participa, íntegra, o como una parte representativa de las mismas, el espacio provincial segoviano 5 (Figura 1).

La Denominación de Origen Ribera de Duero es la más afamada de todas las figuras agroalimentarias de calidad de Castilla y León (Molinero, 2011). Bajo su paraguas de protección se produce una amplia y selecta gama de vinos que van desde los rosados a los, por excelencia, tintos (en sus variantes jóvenes, crianzas, reservas y grandes reservas). Segovia, con cuatro municipios (Aldehorno, Honrubia de la Cuesta, Montejo de la Vega de la Serrezuela y Villaverde de Montejo), participa solamente del $0,8 \%$ de la superficie integrada en la $\mathrm{DO}^{6}$, en mucha menos extensión que la representada por las otras tres provincias "ribereñas", Burgos $(81,7 \%)$, Valladolid $(10 \%)$ y Soria $(7,5 \%)$. De ahí las exiguas cifras que se contemplan dentro de la de por sí ya escasa producción de vino provincial $(36.874 \mathrm{hl}$ en $2012,2,15 \%$ del total regional). Algo mayores son las pertenecientes a la Denominación de Origen Rueda, especializada, en gran medida, en el vino blanco (con el "verdejo" como variedad principal), y que prolongan los predominantes pagos vallisoletanos (en 53 municipios) $\mathrm{y}$, en menor medida, abulenses (4), por términos de Aldeanueva del Codonal,

\footnotetext{
${ }^{4}$ Presente en las ediciones de las más prestigiosas ferias y eventos relacionados con la alimentación y la gastronomía de calidad, como Alimentaria de Barcelona o el Salón Gourmet de Madrid. A este respecto, ha sido presentado meses atrás un amplio catálogo que busca facilitar -con el afán de proyectar- a los cocineros profesionales el conocimiento de los productos "selectos" de la Comunidad ("Tierra de Sabor elabora su vademécum gastronómico”. El Adelantado de Segovia, 13 de marzo de 2014).

5 "Segovia apuesta por la excelencia alimentaria con 15 marcas de calidad". El Adelantado de Segovia, 17 de marzo de 2014. Hay que destacar, también, que como complemento al marketing de Comunidad, la Diputación de Segovia impulsa la marca propia Alimentos de Segovia, con la que se garantiza la calidad y promoción de todos aquellos alimentos que se producen, elaboran o transforman en la provincia y la utilizan como distintivo. Además de los contemplados por Tierra de Sabor, se adscriben a esta enseña provincial otras producciones englobadas en los ámbitos de los derivados lácteos, las legumbres, las mieles o la repostería, entre otras empresas de restauración, carnicerías, mayoristas y distribuidores de alimentación, mataderos y salas de despiece.

${ }^{6}$ Contando con una sola bodega adscrita a la Denominación: la Bodega Aldekoa, que comercializa la marca Pérez Veros, "el ribera del Duero segoviano".
} 
Aldehuela del Codonal, Coca, Codorniz, Donhierro, Fuente de Santa Cruz, Juarros de Voltoya, Montejo de Arévalo, Nava de la Asunción, Nieva, Rapariegos, San Cristobal de la Vega, Santiuste de San Juan Bautista y Tolocirio ${ }^{7}$. Por su parte, otros 9 municipios segovianos -y exclusivamente segovianos- configuran con sus viñedos la mención Vino de Calidad de Valtiendas, con tres bodegas de referencia en el homónimo término (Bodega Tinto Redreja, Bodegas Vagal y Bodega Valviña) donde se producen sus singulares rosados y tintos, elaborados con unos porcentajes mínimos establecidos de variedades autóctonas ("tempranillo" y "albillo") recogidas por las tierras de vides de Aldeasoña, Calabazas de Fuentidueña, Cuevas de Provanco, Fuentesoto, Fuentidueña, Laguna de Contreras, Sacramenia, Torreadrada y Valtiendas. Un panorama el de los vinos y viñedos de calidad al que se incorporan también los asociados a la más genérica mención de Vinos de la Tierra de Castilla y León.

Figura 1. Figuras agroalimentarias de calidad en la provincia de Segovia.

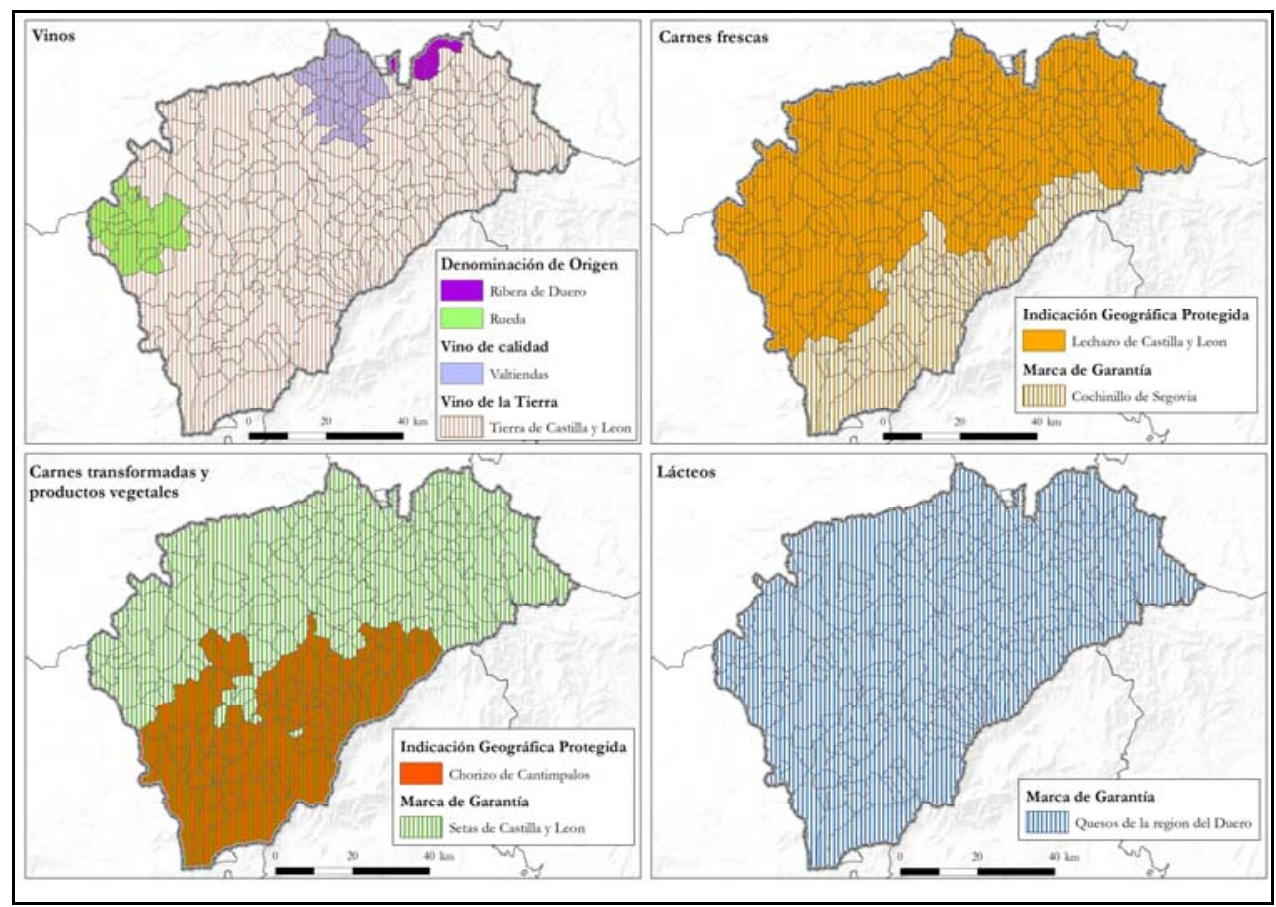

Fuente: Elaboración propia, a partir de ITACyL: Subdirección de Calidad y Promoción Alimentaria.

${ }^{7}$ Con bodegas asociadas a la Denominación en Montejo de Arévalo (Solana Arco Iris), Nieva (Ossian Vides y Vinos y Viñedos de Nieva) y Santiuste de San Juan Bautista (Bodega Cerrosol). 
En el ámbito geográfico de la Cuenca del Duero, se entiende por lechazo a la cría de oveja -macho y hembra- que todavía mama (edad de sacrificio hasta 35 días; de 4,5 a $7 \mathrm{~kg}$ en canal sin cabeza ni asadura). Con procedencia de las razas autóctonas "churra", "castellana" y "ojalada", admitiéndose únicamente los cruces entre ellas, la Indicación Protegida Lechazo de Castilla y León ampara la producción y comercialización de una de las carnes de mayor predicamento en la Comunidad. También en Segovia, provincia afamada por la dedicación ganadera hacia este tipo de orientación y en la que el lechazo asado (al estilo segoviano) y las chuletillas de cordero cobran carta de representación, como platos típicos, por tierras de Cuéllar, Sacramenia, Sepúlveda, Riaza y Pedraza. Lo mismo ocurre, incluso con mayor singularidad dentro del conjunto regional, con el cochinillo, quizás el producto más representativo de la gastronomía segoviana. Como Marca de Garantía, desde 2002, comercializado el producto como fresco o asado, la materia prima son los lechones de raza blanca producidos exclusivamente en la provincia de Segovia. En sus especificaciones la de haber sido alimentados solo con leche materna y la de presentar un peso al sacrificio que no exceda los 6,5 kilogramos (en una edad máxima de tres semanas).

Ligada también a la sobresaliente producción porcina provincial, la Indicación Geográfica Protegida Chorizo de Cantimpalos tiene en la carne fresca del cerdo graso su razón de ser. En su prístina elaboración juegan un papel esencial de aderezo la sal y el pimentón, como ingredientes básicos, a los que también se les suele añadir ajo y orégano, siendo sometido el producto final a un proceso de secado y maduración, y dando lugar a las afamadas variantes de "sarta", "achorizado" y "cular". La zona de elaboración comprende a los municipios localizados en el tercio surocciental de la provincia: Abades, Adrada de Pirón, Aldealengua de Pedraza, Arahuetes, Armuña, Besardilla, Bercial, Bernardos, Bernuy de Porreros, Brieva, Caballar, Cabañas de Polendos, Cantimpalos, Carbonero el Mayor, Casla, Collado Hermoso, Cubillo, Encinillas, El Espinar, Escobar de Polendos, Espirdo, Gallegos, Garcillán, Ituero y Lama, Juarros de Riomoros, La Lastrilla, La Losa, La Matilla, Labajos, Lastras del Pozo, Marazoleja, Marazuela, Martín Miguel, Marugán, Matabuena, Monterrubio, Muñopedro, Navafría, Navas de San Antonio, Orejana, Ortigosa del Monte, Otero de Herreros, Palazuelos de Eresma, Pedraza, Pelayos del Arroyo, Prádena, Rebollo, Roda de Eresma, Sangarcía, Santa María la Real de Nieva, Santiuste de Pedraza, Santo Domingo de Pirón, Segovia, Sotosalbos, Tabanera la Luenga, Torrecaballeros, Torreiglesias, Torre Val de San Pedro, Trescasas, Turégano, Valdeprados, Valleruela de Pedraza, Valleruela de Sepúlveda, Valseca, Valverde del Majano, Vegas de Matute, Ventosilla y Tejadilla, Villacastín y Zarzuela del Monte ${ }^{8}$.

${ }^{8}$ Con industrias elaboradoras en Villacastín (2), La Lastrilla, La Losa, Bernuy de Porreros (2), Cantimpalos (8), Valseca, Carbonero el Mayor y Tabanera la Luenga. 
Tabla 2. Distribución de la superficie de agricultura ecológica en la provincia de Segovia.

\begin{tabular}{|l|r|r|}
\multicolumn{1}{|c|}{ Tipo de cultivo } & No explotaciones & ha \\
\cline { 2 - 3 } Prados o praderas permanentes & 7 & 449 \\
\hline Cereales & 15 & 300 \\
\hline Oleaginosas & 10 & 126 \\
\hline Leguminosas & 12 & 80 \\
\hline Uva de vinificación & 5 & 86 \\
\hline Patatas & 4 & 10 \\
\hline Hortalizas & 5 & 5 \\
\hline Otros cultivos & 7 & 52 \\
\hline Total agricultura ecológica & 28 & 1.108 \\
\hline
\end{tabular}

Fuente: INE: Censo Agrario, 2009.

Dentro del Distintivo de calidad alimentaria Tierra de Sabor, en el apartado de otras figuras de calidad aparecen las de Agricultura Ecológica de Castilla y León y Producción Integrada de Castilla y León. La primera hace referencia al conjunto de prácticas agrarias, según define la reglamentación comunitaria (Reglamento CEE n ${ }^{\circ}$ 2092/91), que siguen indicaciones al método ecológico de la producción, recibiéndose con ello un complemento extra de "subvención". Son productos agrícolas vegetales o animales no transformados, productos agrícolas vegetales transformados, destinados a la alimentación humana y preparados básicamente a partir de uno o más ingredientes de origen vegetal o animal, y los alimentos para animales, caso de los piensos compuestos o materias primas para su alimentación. El Consejo de Agricultura Ecológica de Castilla y León (CAECyL) es el órgano de la administración que controla las zonas de producción, que serán aquellas consideradas adecuadas para la obtención de productos agrícolas y ganaderos de acuerdo con los estándares ecológicos y criterios de calidad (cumplimiento de unas condiciones ambientales mínimas, rotación adecuada de cultivos, planificación del laboreo del suelo en base a las normas del Consejo, control de plagas y enfermedades por medio de técnicas preventivas y tratamientos autorizados con productos de origen natural, control de adventicias y malas hiervas mediante laboreo adecuado). Según el Censo Agrario de 2009, en Segovia había 28 explotaciones donde se practicaba agricultura ecológica certificada (calificada o en proceso de conversión), dando un total de 1.108 ha (Tabla 2). Las producciones más importantes tenían que ver, en mayor o menor medida, con la alimentación animal. Destacándose, también, la relativa presencia de este tipo de 
producción en patatas, hortalizas y uva de vinificación ${ }^{9}$. Así como 6 explotaciones ganaderas (330 cabezas de bovino) en las que primaba la extensificación ambiental. Con todo, la agricultura ecológica es todavía minoritaria dentro del espacio agrario provincial, si bien es percibida, ya, como un tipo singularizado de explotación (Tabla $3)$.

Tabla 3. Percepción sobre la importancia de la agricultura ecológica en la provincia de Segovia.

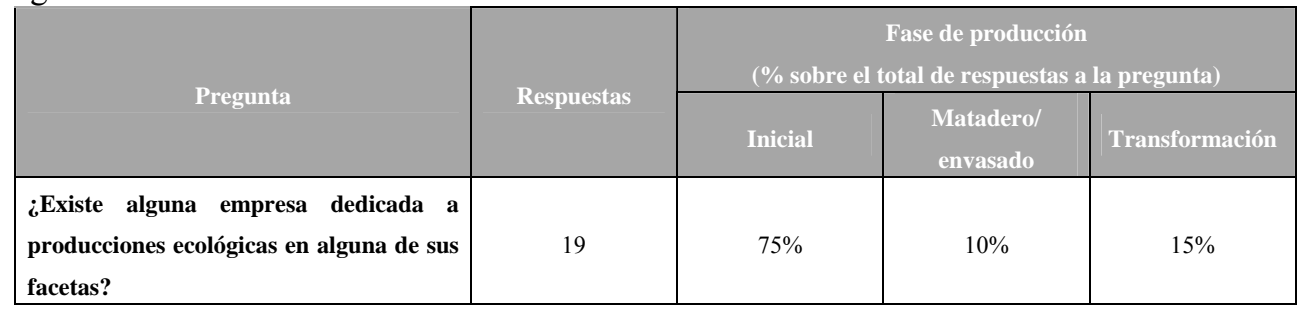

Fuente: Departamento de Geografía de la Universidad de Valladolid: Encuesta a los Municipios de Segovia, 2014.

Por su parte, la Producción Integrada de Castilla y León es otro elemento de calidad indiscutible, merced a las prácticas y exigencias impuestas a los productores en aras a la consecución de productos controlados y de confianza, por mor del mayor esfuerzo del agricultor. Se trata de un método de producción agrícola que obtiene productos de calidad diferenciada, garantizando la seguridad alimentaria para el consumidor, protegiendo el medio ambiente a través de la utilización racional de los medios de producción (semillas, abonos, maquinaria) e identificando las fases de producción y seguimiento del producto, desde la parcela de origen hasta el consumidor final (trazabilidad). Comprende cultivos de zanahoria, patata, frutales de pepita, maíz dulce, remolacha de mesa, viñedo, lechuga, puerro, ajo, cebolla, cereal y cerezo $^{10}$.

${ }^{9}$ Empresas segovianas asociadas, actualmente, con el distintivo Agricultura Ecológica de Castilla y León son las porcineras Acompor-Pigs y Carnipor, en Vallelado, las chacineras Bernardino Postigo e Hijos, en Cantimpalos, Embutidos El Enebral, en El Arenal, y Fábrica de Embutidos La Prudencia, en Villacastín, así como las cárnicas Las Hoces, en Sepúlveda, y Ma Cruz Bravo Benito, en La Losa, o las hortícolas Huerta Castellana, Las Lagunas de Sanchonuño y Ultracongelados del Duero, en Sanchonuño.

${ }^{10}$ En la provincia de Segovia, empresas hortícolas asociadas a la Producción Integrada, controlando la calidad, el origen y la trazabilidad de todos los procesos de producción y comercialización, son: Huerta Castellana, La Flor del Carracillo y Lagunas de Sanchonuño, en Sanchonuño, Sociedad Cooperativa Comarca de Vallelado, en Mata de Cuéllar, Sociedad Cooperativa del Campo GLUS-I, en Cuéllar, Sociedad Cooperativa El Pinar del Carracillo, en Gomezserracín, y Cándido Muñoz Pascual, en Carbonero el Mayor. 
En suma, se puede afirmar que la actividad agraria del futuro ha de basarse en todos estos distintivos, figuras y "marcas" de calidad, apostando por el control y la trazabilidad, el conocimiento del origen de las producciones, la conservación de las variedades más genuinas, la sostenibilidad ambiental y el cuidado en los métodos de elaboración. La calidad agroalimentaria es, por encima de cualquier otra consideración, reto y oportunidad para el desarrollo rural.

\section{LA DIVERSIFICACIÓN ECONÓMICA Y FUNCIONAL: ESTRATEGIAS Y LIMITACIONES PARA EL DESARROLLO RURAL SEGOVIANO}

A lo largo de las últimas décadas, el mundo rural segoviano, como, en general, todo el mundo rural castellano y leonés y español, ha tenido que soportar intensos cambios socioeconómicos y funcionales que han provocado un aumento de los desequilibrios existentes en su seno (Maya e Hidalgo, 2009). Así, el espacio rural de la provincia sigue siendo mayoritariamente agrario, desde el punto de vista de los usos del suelo y de los paisajes dominantes, pero mientras unos ámbitos se adaptan bien a la nueva dialéctica del funcionamiento urbano-rural, otros sufren un proceso de descualificación y despoblamiento, pues la especialización, tecnificación y, con ello, modernización de las estructuras agrarias ha motivado que en estos no se pueda crear empleo en la agricultura o la ganadería, sino todo lo contrario.

En el marco del "posproductivismo" imperante, son recurrentes las proclamas a la diversificación y a la pluriactividad como garantes inexcusables del desarrollo territorial (Maya, 2008). Del progreso de unas áreas que, además, han dejado de ser predominantemente agropecuarias (como lo demuestran, a escala provincial, las cifras absolutas y los porcentajes de distribución sectorial del empleo por tipo de municipio -Tabla 4 y Figura 2-). La actividad agraria, por consiguiente, no puede ser la piedra angular, en exclusiva, del desarrollo rural (Molinero y Alario, 1994). Por más que sí lo sea, en su dimensión de desarrollo agrario -o agroalimentario en base a las manufacturas-, encontrando recurso y acomodo en todas esas producciones amparadas por las figuras de calidad, los productos ecológicos e integrados, el mantenimiento y cuidado del paisaje y, por supuesto, a partir de toda otra serie de producciones competitivas donde la provincia cobra carta de entidad: como las hortícolas o la sobresaliente ganadería industrial (Martínez, 2014). La adaptación del medio rural hacia la pluriactividad coincide, como señalan reputados estudiosos, en una apuesta decidida por las actividades turísticas, residenciales y de ocio (Moltó y Hernández, 2004); de tal forma que los espacios rurales, en sentido amplio, y algunos de los segovianos, en particular, "emergen" actualmente -y no tan actualmente- como el soporte físico para cubrir las necesidades de ocio y esparcimiento de la que se ha venido en denominar como sociedad urbanizada (Ortega, 2004). 
Tabla 4. Distribución sectorial del empleo por tipo de municipio en Segovia.

\begin{tabular}{|l|r|r|r|r|r|}
\cline { 2 - 6 } \multicolumn{1}{c|}{ Tipo de municipio } & \multicolumn{1}{c|}{ Agrario } & Industrial & Construcción & \multicolumn{1}{c|}{ Servicios } & Total \\
\hline Capital & 352 & 833 & 1.214 & 21.399 & 23.798 \\
\hline $\begin{array}{l}\text { Periurbano y áreas de influencia } \\
\text { urbana }\end{array}$ & 341 & 1.321 & 656 & 3.486 & 5.804 \\
\hline Centros de servicios & 1.600 & 2.445 & 1.368 & 6.873 & 12.286 \\
\hline Municipios rurales & 4.274 & 1.758 & 1.157 & 4.870 & 12.059 \\
\hline
\end{tabular}

Fuente: Ministerio de Empleo y Seguridad Social ${ }^{11}$.

Figura 2. Porcentaje de ocupados en la actividad agraria por tipo de municipio en Segovia.

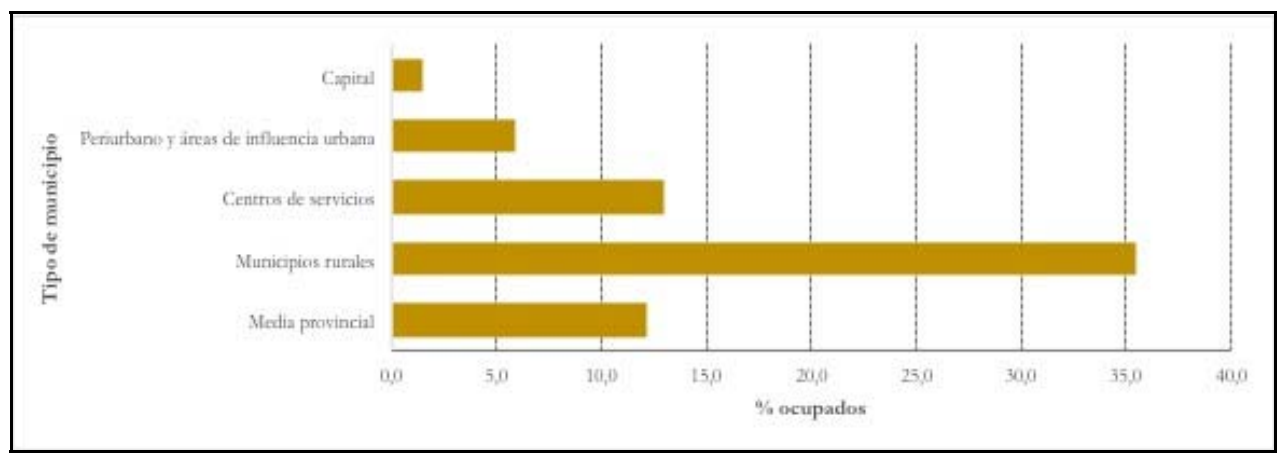

Fuente: Elaboración propia. Ministerio de Empleo y Seguridad Social ${ }^{12}$.

No se puede pensar, sin embargo, que el "posproductivismo", así entendido, sea la solución a los problemas que aquejan a gran parte del espacio rural de Castilla y León -y por ende, se podría colegir, al de Segovia- ${ }^{13}$; el de los términos municipales de menor tamaño demográfico, los que en mayor medida, también, han quedado al margen de la modernización y el desarrollo agrario. Ya que, aunque los agricultores y ganaderos cada vez son menos en el panorama social del campo, todavía tienen mucho peso (como se evidencia en la figura adjunta), especialmente en los pueblos del "rural profundo" (los municipios de menos de 2.000 habitantes), donde hay todavía un tercio aproximado de ocupados agrarios.

${ }^{11}$ Explotación estadística de Datos de Afiliación de Trabajadores al Sistema de la Seguridad Social por régimen, municipio y actividad (CNAE-09), a diciembre de 2013.

${ }^{12}$ Explotación estadística de Datos de Afiliación de Trabajadores al Sistema de la Seguridad Social por régimen, municipio y actividad (CNAE-09), a diciembre de 2013.

${ }_{13}$ Una interesante reflexión sobre las limitaciones al desarrollo posproductivista y las incertidumbres que se ciernen sobre el medio rural de Castilla y León: la marcha hacia el "cuarto paradigma de desarrollo rural", puede encontrarse en los escritos más recientes de Fernando Molinero Hernando (Molinero, 2012). 
Tabla 5. Desarrollo rural: otras actividades complementarias de la explotación en Segovia.

\begin{tabular}{|l|r|} 
& No explotaciones \\
\hline Turismo, alojamiento y otras actividades recreativas & 36 \\
\hline Artesanía & 6 \\
\hline Transformación de productos agrícolas (elaboración de embutidos, queso, vino, ...) & 22 \\
\hline Producción de energía renovable para la venta (eólica, biogás, solar, ...) & 11 \\
\hline Transformación de la madera (aserrado) & 4 \\
\hline Acuicultura (cría de peces, cangrejos, ranas, ...) & 4 \\
\hline Trabajos agrícolas bajo contrato para otras explotaciones & 86 \\
\hline Trabajos no agrícolas bajo contrato & 14 \\
\hline Silvicultura & 10 \\
\hline Otras & 60 \\
\hline Total de explotaciones & 224 \\
\hline & \\
\hline
\end{tabular}

Fuente: INE: Censo Agrario, 2009.

Tabla 6. Medidas de desarrollo rural: explotaciones que se han beneficiado de alguna medida en los últimos tres años en la provincia de Segovia.

\begin{tabular}{|l|r|} 
& \multicolumn{1}{|c|}{ Medida } \\
\hline Utilización de los servicios de asesoramiento & 48 \\
\hline Modernización de explotaciones agrícolas & 47 \\
\hline Aumento del valor añadido de los productos agrícolas y forestales & 13 \\
\hline Pagos vinculados a la Directiva relativa al marco del agua & 1 \\
\hline Pagos relacionados con la agricultura ecológica & 2 \\
\hline Pagos relacionados con otras ayudas agroambientales & 237 \\
\hline Ayudas relativas al bienestar de los animales & 45 \\
\hline
\end{tabular}

Fuente: INE: Censo Agrario, 2009.

Esta circunstancia, prosiguiendo con las reflexiones del autor que acaba de ser citado, lleva a pensar que el resto de actividades -las industriales y los servicios- son muy escasas en la mayoría de los casos o están polarizadas en algunos núcleos centros y cabeceras- o territorios concretos -con potencialidad y atractivo para el ocio, la residencia secundaria y el turismo-.

En cualquier caso, y aunque poco, los agricultores y ganaderos segovianos han tendido a diversificar sus funciones para adaptarse a los nuevos tiempos, comenzando a tener alguna representación las explotaciones acogidas a las medidas de desarrollo rural $y$ al fomento de otras actividades -no agrarias- complementarias de la 
explotación ${ }^{14}$. De las 8.863 explotaciones registradas en el Censo Agrario de 2009, 224 (el 2,53\%) se han acogido a la línea priorizada de diversificación de actividades (Tabla 5). Una cuantía muy similar a la que corresponde con la de las distintas unidades de producción beneficiadas de alguna medida de desarrollo rural, en particular las derivadas de la protección del medio ambiente ${ }^{15}$ (Tabla 6).

No obstante, mayor difusión de resultados, en lo concerniente a la diversificación de actividades económicas, han tenido los programas de desarrollo rural ${ }^{16}$. A día de hoy, cuatro Grupos de Acción Local (GAL) son los encargados de la gestión, el control de fondos (públicos y privados) y la toma de decisiones sobre los proyectos a desarrollar dentro de los marcos geográfícos de referencia (espacios de cooperación y desarrollo territorial) establecidos al amparo y salvaguarda de la Iniciativa

14 La PAC tiene dos vertientes -“pilares"- diferenciadas: las ayudas directas en apoyo compensatorio al declive de las rentas agrarias, por una parte, y el desarrollo rural, con cada vez más relevancia en el conjunto. La regulación de mercados y la reforma de estructuras, como se convenía hace años en resaltar. La gran reforma de 1992 avanzó en la línea de combinar ajuste y reforma, conjugando la finalidad de lograr una actividad agraria competitiva con el mantenimiento de las áreas rurales. Desde el punto de vista del desarrollo rural, las preocupaciones por los temas ambientales y otros problemas estructurales del campo inspiraron las llamadas "medidas de acompañamiento": modernización, rejuvenecimiento y mejora de las explotaciones, formación, medidas agroambientales,... Con la llamada Agenda 2000, se hace evidente el compromiso de la Comunidad (UE) de mantener unas poblaciones rurales vivas y de conservar el patrimonio rural europeo. En este sentido, aun reconociendo la importancia de unas actividades agrarias fuertes en la economía rural, las recomendaciones de la "nueva" PAC señalaban que las comunidades rurales no debían de depender en el futuro exclusivamente de éstas, siendo necesario el establecimiento de un marco coherente y sostenible que refuerce el tejido económico y social de las zonas rurales y reduzca su continuada despoblación (Baraja et al., 2001). Entre las medidas del catálogo de desarrollo rural se contemplan algunas de acompañamiento a la política de los pagos directos y compensación de rentas y otras tendentes a la promoción de la diversidad funcional y pluriactividad en el medio rural.

${ }^{15}$ Las medidas agroambientales prestan apoyo adicional a las explotaciones en su esfuerzo de proteger la naturaleza con métodos de producción menos intensos, gestión sostenible de los recursos y fomento de la biodiversidad.

${ }^{16}$ Instrumentos claves de la Política Regional de la Unión Europea desde la reforma de los Fondos Estructurales de 1988. La Iniciativa comunitaria LEADER (como los programas nacionales PRODER y PRODERCAL, desarrollados en el marco de financiación de las regiones de Objetivo 1 hasta 2006, en las áreas excluidas de la anterior) en sus diferentes formulaciones, LEADER I (no presente en la provincia), LEADER II (el de Codinse, 19952000), LEADER + (Codinse y Aidescom, 2001-2006) y LEADERCAL (Codinse, Aidescom, Honorse y Segovia sur, 2007-2013), es concebida como un instrumento para el desarrollo socioeconómico de las áreas rurales desfavorecidas de Europa, a partir de un enfoque territorial, ascendente e integrado de cooperación entre los diferentes agentes implicados (agentes locales, económicos, sociales, políticos y los técnicos responsables del asesoramiento) y con la mirada puesta en la diversificación económica con la que abordar las problemáticas de declive productivo y social que venían acentuándose desde la crisis de la actividad agraria tradicional. Un repaso a la política de desarrollo rural en Castilla y León puede hacerse a partir de Alario y Baraja (2006) y Fernández (2011). 
LEADERCAL para la provincia de Segovia (Figura 3). A partir de ellos, el diseño de las estrategias y el enfoque de las capacidades para el progreso se basan en la articulación de los sectores más significativos de la economía y sociedad local, previo conocimiento de las necesidades y las potencialidades del entorno, con la finalidad de posicionar los territorios-acción, apoyando las iniciativas productivas más o menos innovadoras y con proyección y siendo el escenario para la territorialización de las diferentes políticas sectoriales, con el afán puesto en la plurifuncionalidad ${ }^{17}$.

El progreso de la diversificación de funciones en el espacio rural segoviano ha tenido en el turismo, como ha sido práctica habitual en el conjunto de ámbitos para el desarrollo rural auspiciados por los diferentes programas contemplados desde los años noventa, uno de los baluartes sobre los que soportar la readaptación funcional de las áreas campesinas a partir de la creciente desagrarización y consecuente búsqueda de alternativas en la terciarización del campo (Alario, 2004). Varios son los factores convencionalmente esgrimidos para explicar esta realidad: en primer lugar, la necesidad de promover nuevas actividades económicas que permitieran el mantenimiento de la población rural -cada vez menos agraria- capaces de generar unas rentas complementarias y/o alternativas a las tradicionales; en segundo lugar, la apuesta decidida por la financiación, desarrollo y promoción de infraestructuras de alojamiento de la mano de las iniciativas LEADER y PRODER; y, en tercer lugar, el cambio operado en el modelo turístico español, con la promoción de nuevos productos -a añadir a los masificados y estacionalizados del sol y la playa o de nievecon los que dar respuesta a la demanda solvente de una sociedad, eminentemente urbanizada, que busca en el contacto con la naturaleza, los valores del paisaje y la recuperación del patrimonio agrario y cultural, nuevas fórmulas con las que satisfacer las necesidades de ocio y esparcimiento (Butler, 1998; Crouch, 2006).

\footnotetext{
${ }^{17}$ Un simple vistazo a la rica información referida a los GAL, y que puede ser fácilmente consultada en las correspondientes páginas web, da buena cuenta de cuáles son las orientaciones, las actuaciones concretas y las líneas estratégicas con las que afrontan el desarrollo integral de los respectivos territorios-acción. Consultar: www.codinse.com (Coordinadora para el Desarrollo Integral del Nordeste de Segovia); www.aidescom.com (Asociación Intermunicipal para el Desarrollo Local en la Comarca de Santa María la Real de Nieva); www.segoviasur.com (Asociación para el Desarrollo Rural Segovia Sur); y www.tierra depinares.es (Horizonte Norte Segoviano-Tierra de Pinares). En la situación presente, en la que los recortes paralizan las inversiones económicas de los GAL -que han cumplido su función y deben continuar haciéndolo-, se hace necesaria la búsqueda de alternativas de financiación con las que seguir impulsando el desarrollo rural. Así, por ejemplo, la ADE Rural y la Diputación de Segovia, a través de su marca de territorio Segovia Experience Empresas, han comenzado a sentar las bases de trabajo para la incentivación del emprendimiento en el medio rural ("La ADE Rural y las Diputaciones trabajarán en común para la promoción del empleo en los pueblos". El Norte de Castilla, 13 de febrero de 2014).
} 
Figura 3. Grupos de Acción Local (GAL) que operan en la provincia de Segovia, 2007-2013.

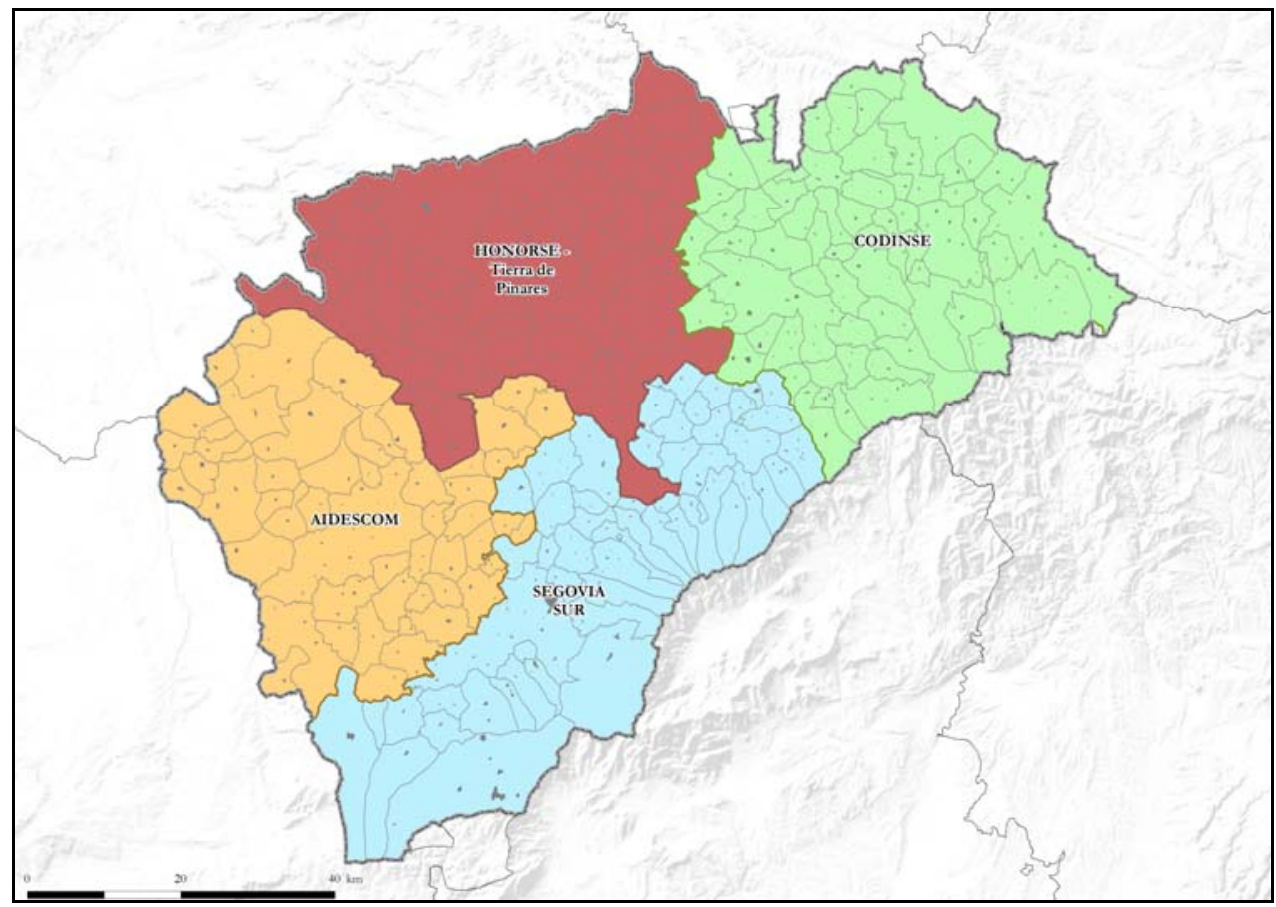

Fuente: Elaboración propia, a partir de JCyL: Servicio de Iniciativas y Promoción del Desarrollo de la Consejería de Agricultura y Ganadería.

Actualmente, todos los Grupos de Acción Local prosiguen en la línea de afianzar por la vía del turismo rural la dinamización económica de sus territorios de acción; sin embargo, las actuaciones más recientes tienden -más que a continuar fomentando la infraestructura de alojamientos- a la promoción de actividades complementarias (deportivas, educativas ${ }^{18}$, culturales, paisajísticas, gastronómicas) y la mejora de la comercialización de los productos con la finalidad de atraer el mayor número posible de visitantes, con especial interés por los segmentos más cualificados (difusión y

\footnotetext{
${ }^{18}$ Por la repercusión que está llamado a tener en la dinamización de un pequeño municipio rural, se destaca la reciente inauguración del Aula de la Naturaleza de Duruelo, que revitalizará todo el Nordeste en palabras de sus promotores (Ayuntamiento, CODINSE y Colegios El Valle, de Madrid, que ha llevado a cabo la inversión y se ocupará de la explotación ("El Ayuntamiento inaugura un Aula de la Naturaleza para escolares que creará medio centenar de empleos". El Adelantado de Segovia, 13 de marzo de 2014).
} 
publicidad, campañas de imagen de "marca", apuesta por la calidad y la singularidad) ${ }^{19}$.

Tabla 7. Percepción sobre la importancia de los programas de desarrollo rural en la provincia de Segovia.

\begin{tabular}{|c|c|c|c|c|c|c|}
\hline \multirow[t]{2}{*}{ Pregunta } & \multicolumn{3}{|c|}{$\%$ sobre los 121 municipios encuestados } & \multicolumn{3}{|c|}{$\begin{array}{l}\% \text { sobre el total de respuestas a la } \\
\text { pregunta }\end{array}$} \\
\hline & SI & NO & $\%$ & SI & NO & $\%$ \\
\hline $\begin{array}{l}\text { ¿Se ha creado empleo gracias } \\
\text { a las ayudas ofertadas por los } \\
\text { programas e iniciativas de } \\
\text { desarrollo rural? }\end{array}$ & $21,07 \%$ & $58,26 \%$ & $79,33 \%$ & $26,56 \%$ & $73,44 \%$ & $100 \%$ \\
\hline \multirow[b]{2}{*}{ Pregunta } & \multicolumn{6}{|c|}{$\%$ sobre el total de respuestas afirmativas a la pregunta } \\
\hline & $\begin{array}{l}\text { Ind. } \\
\text { alimentaria }\end{array}$ & $\begin{array}{l}\text { Otras } \\
\text { industrias }\end{array}$ & \multicolumn{2}{|c|}{ Turismo rural } & \multicolumn{2}{|c|}{ Servicios en general } \\
\hline ¿Ha creado empleo en...? & $14,75 \%$ & $14,75 \%$ & \multicolumn{2}{|c|}{$29,52 \%$} & \multicolumn{2}{|c|}{$40,98 \%$} \\
\hline
\end{tabular}

Fuente: Departamento de Geografía de la Universidad de Valladolid: Encuesta a los Municipios de Segovia, 2014.

A pesar de ello, y aunque es evidente que el turismo rural ha generado riqueza y diversidad económica en la provincia, son muchos los ámbitos rurales en los que dudosamente es esa actividad capaz de ser alternativa a la agricultura y ganadería tradicional (Delgado, 2014). Primero, porque los trabajadores directamente empleados en el sector son relativamente pocos; segundo, porque los municipios del "rural profundo" apenas cuentan con este tipo de ocupación (como lo corroboran las respuestas dadas, mayormente por ellos, al ser preguntados los Ayuntamientos por la percepción que tienen de la importancia de las iniciativas de desarrollo rural y, particularmente, sobre la capacidad de creación de empleo por parte del turismo rural -Tabla 7-); y, tercero, porque es evidente que las áreas verdaderamente turísticas coinciden con unos pocos espacios que son los que concentran los factores de

${ }^{19}$ Ello se ha traducido en el esfuerzo por promocionar las áreas turísticas del espacio rural provincial, que ni con mucho agotan todo el potencial turístico atesorado por Segovia, y a las que el Patronato Provincial de Turismo dedica una atención especial (ver: www.segoviaturismo.es), trabajando por hacer de Segovia uno de los destinos predilectos a escala nacional ("Segovia fue el quinto destino español de turismo rural en octubre". El Adelantado de Segovia, 3 de diciembre de 2013). O en la apuesta decidida por la calidad, como el Nuevo Decreto de Turismo Rural de Castilla y León, en vigor desde el pasado 3 de marzo de 2014, tendente a regularizar la categorización de los establecimientos, viene a confirmar. 
atracción: la Sierra, en su conjunto, y los espacios significados por su riqueza ambiental $^{20}$.

Empero, la oferta de ocio rural para la población urbana no se centra en exclusiva ni es tan relativamente novedosa como la que representa el turismo rural. La impronta de la población no residente en los municipios rurales, bien sea a través del fenómeno generalizado de la población "vinculada" o el auge que ha tenido -y tiene- la producción de espacios residenciales y de ocio vinculados a la aglomeración madrileña, en los ámbitos del mediodía serrano, hace que se pueda hablar de un cierto "renacimiento" rural, si bien es cierto que este quedaría circunscrito a las estancias estacionales de la población no empadronada, los periodos festivos y de vacaciones, en los que casas familiares o residencias secundarias cobran vida y el bullicio de los núcleos y las urbanizaciones carta de entidad. Una realidad, que ha sido estudiada con la atención que merece a escala provincial y a la del conjunto de la comunidad autónoma de Castilla y León, como una dimensión más del análisis del modelo de poblamiento provincial y regional, que trasciende a lo económico, al ser un elemento sustancial para el sostenimiento de las haciendas, los negocios y los servicios locales (Molina y Delgado, 2014; Delgado y Martínez, 2014). Un "renacimiento" cíclico que no por efímero y circunstancial ha de ser desdeñado de las diferentes propuestas de desarrollo territorial.

Tabla 8. Distribución de las empresas industriales por tipo de municipio en Segovia.

\begin{tabular}{|l|r|r|}
\hline \multicolumn{1}{|c|}{ Tipo de municipio } & Empresas agroindustriales & Total de empresas industriales \\
\cline { 2 - 4 } Capital & 23 & 105 \\
\hline Periurbano y áreas de influencia urbana & 49 & 106 \\
\hline Centros de servicios & 63 & 175 \\
\hline Municipios rurales & 76 & 156 \\
\hline Total provincial & 211 & 542 \\
\hline
\end{tabular}

Fuente: Ministerio de Empleo y Seguridad Social ${ }^{21}$.

${ }^{20}$ Las referencias a estos tipos de espacios son una constante cuando se quiere alzaprimar su condición de destinos sobresalientes del turismo rural (Martín et al., 2003). En Segovia ha sido estudiado este hecho por Hortelano (2012) para la Sierra de Ayllón, Sanz (2006) para las Hoces del Río Duratón y Martín y Martín (2014) para toda la comarca del Nordeste Segoviano. Frecuentes llamadas de atención sobre el mismo hecho: "El Parque Nacional del Guadarrama busca impulsar el turismo en su entorno". El Norte de Castilla, 28 de noviembre de 2013; "Aumentan un 6,3\% las visitas a la red de espacios naturales y casas del parque en Segovia". El Adelantado de Segovia, 6 de febrero de 2014.

${ }^{21}$ Explotación estadística de Datos de Afiliación de Trabajadores al Sistema de la Seguridad Social por régimen, municipio y actividad (CNAE-09), a diciembre de 2013. 
Tabla 9. Distribución de las industrias agroalimentarias por tipo de municipio en Segovia.

\begin{tabular}{|c|c|c|c|c|c|c|c|}
\hline Tipo de municipio & Cárnicas & Hortícolas & $\begin{array}{l}\text { Vinícolas/ } \\
\text { bebidas }\end{array}$ & $\begin{array}{l}\text { Derivad. } \\
\text { cereales }\end{array}$ & $\begin{array}{l}\text { Aliment. } \\
\text { animal }\end{array}$ & Otras & Total \\
\hline Capital & 22 & 0 & 1 & 11 & 2 & 3 & 39 \\
\hline Áreas periurbanas & 28 & 0 & 2 & 4 & 3 & 1 & 38 \\
\hline Centros de servicios & 30 & 11 & 4 & 33 & 4 & 4 & 86 \\
\hline Municipios rurales & 30 & 32 & 13 & 40 & 20 & 13 & 148 \\
\hline Total provincial & 110 & 43 & 20 & 88 & 29 & 21 & 311 \\
\hline
\end{tabular}

Fuente: ITACyL: Agenda de contactos.

Figura 4. Localización de la industria agroalimentaria en la provincia de Segovia.

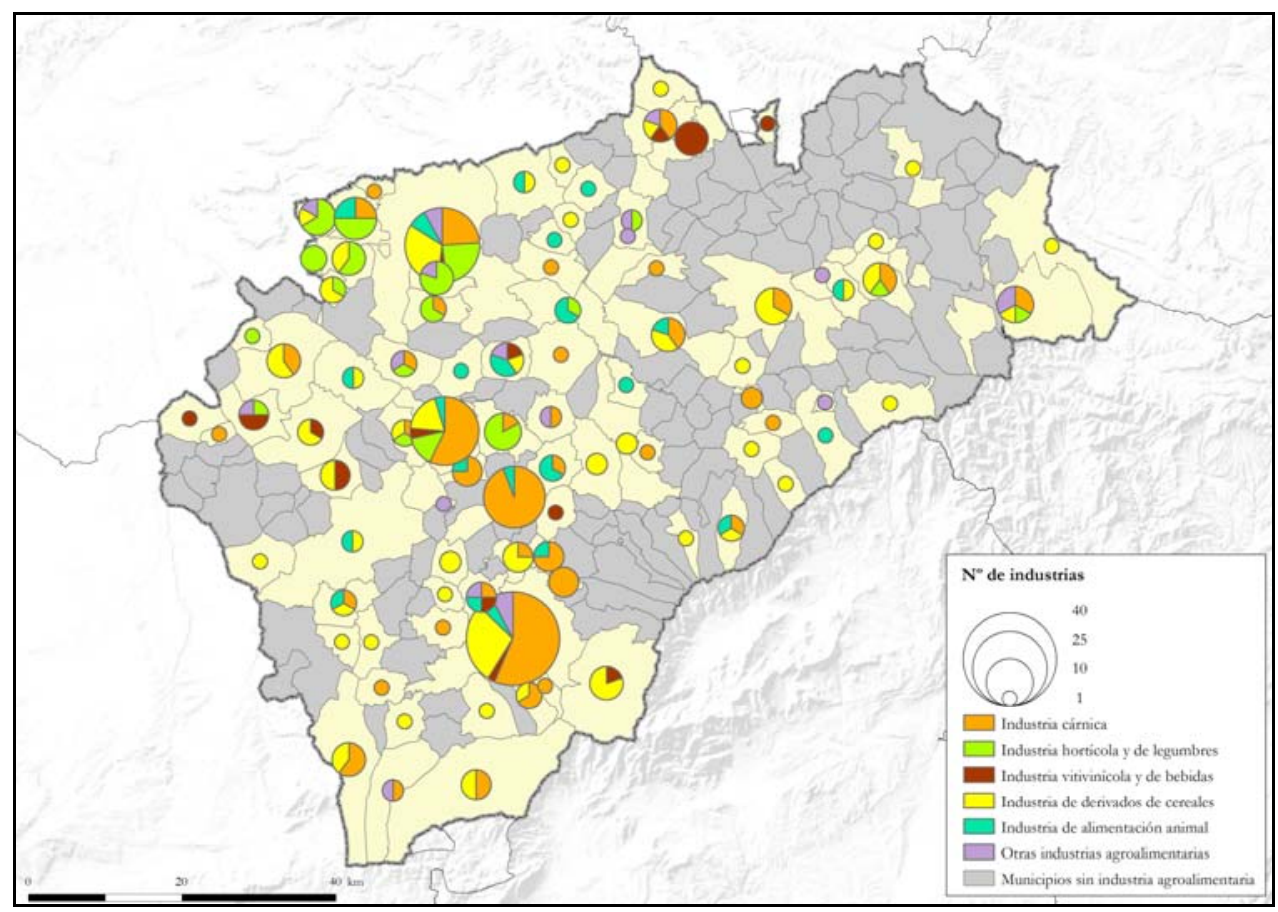

Fuente: Elaboración propia, a partir de ITACyL: Agenda de contactos.

Como tampoco el espacio rural ha de ser ajeno a la presencia, notable por la representación que adquiere dentro del conjunto de la provincia, del aparato industrial (la localización de la industria fuera de la ciudad y del área de influencia urbana alcanza el 61,07\% de los establecimientos) (Tabla 8); y, muy especialmente, de aquellas manufacturas que lo vinculan con la actividad agraria, esto es, la manipulación y transformación de la producción agropecuaria $(40,77 \%$ del total de empresas manufactureras) (Tabla 9). En efecto, Segovia no es una provincia donde 
domine la industrialización, es, por detrás de Zamora y Ávila (y de Salamanca por porcentaje de ocupación laboral), el espacio provincial de la comunidad autónoma de Castilla y León menos industrializado. Con todo, es interesante resaltar la importancia y significado que para el desarrollo rural tiene la industria agroalimentaria, tanto por su fortísima ligazón, como se deduce de lo expuesto con anterioridad, con los procesos de especialización, cualificación y modernización de la actividad agraria, como por su relevancia territorial, al estar presente en un número muy considerable de términos municipales (Figura 4). Ámbitos en los que resulta esencial como motor socioeconómico, y no únicamente por la actividad directa o el empleo que pudiera generar, sino también porque es un factor esencial para el desarrollo agrario y la viabilidad de buen número de explotaciones y de producciones, que, a pesar de las proclamas por la desagrarización y terciarización del campo, siguen siendo lo sustancial del difícil mantenimiento -al menos del "profundo"- mundo rural segoviano.

La industria agroalimentaria está compuesta, fundamentalmente, por manufacturas de primera transformación, estrechamente machiembradas a esa producción agropecuaria y por lo tanto al espacio rural, formando parte esencial de los sistemas productivos locales en base al aprovechamiento del potencial endógeno, y con origen, mayoritariamente, en iniciativas nacientes en el propio entorno ${ }^{22}$. Pequeñas empresas industriales y buen número de cooperativas dan cauce a esta integración vertical de los procesos productivos, acrecentándose, en definitiva, las cotas de valor añadido dejadas por las producciones agrícolas y ganaderas. Firmas, muchas de ellas ya citadas en páginas precedentes, han consolidado al sector, destacando en ramas tan queridas por la especialización agraria provincial como las industrias cárnicas (porcino, aviar, chacineras), las hortícolas, las de los derivados de cereales y alimentación animal y las de bebidas (vinícolas, aguas, destilerías), entre otras de menor representatividad.

\footnotetext{
${ }^{22}$ Entre las empresas industriales dedicadas a la agroalimentación que escapan a este carácter cooperativo o de pequeña empresa familiar son de destacar: Dibaq Diproteg S.A., especializada en alimentación y nutrición animal, con plantas en Fuentepelayo; el Grupo SIRO, con una fábrica de bollería y un centro de I+D+i en El Espinar; Agua Mineral Bezoya, perteneciente al Grupo PASCUAL, con el manantial y la planta envasadora en Ortigosa del Monte; o Destilerías y Crianzas del Whisky (DyC), en Palazuelos de Eresma y Valverde del Majano, de notable actualidad por entrar en las operaciones empresariales de potentes multinacionales del sector ("La UE da luz verde a la compra del Grupo Beam por parte de la japonesa Suntory”. El Norte de Castilla, 24 de abril de 2014).
} 


\section{EPÍLOGO}

Ante este panorama que se acaba de plantear, el espacio rural de Segovia camina tímidamente hacia la diversificación económica, al tiempo que es reflejo de una más que evidente evolución funcional. En él, la sociedad activa -que no es por otra parte la que predomina en los ámbitos sumamente envejecidos y despoblados- está dejando de ser ocupacionalmente agraria para pasar a depender de los servicios. Unos servicios que más allá de los que se derivan del turismo, como fórmula más representativa con la que se ha pretendido operar en desarrollo rural, tienen que ver sobremanera con los de atención a la población. Los empleos en los servicios sociales básicos y en los de asistencia personal son la clave de la terciarización (Delgado 2014). Estos son los límites y las oportunidades que se ciernen sobre el espacio rural segoviano. La modernización de las explotaciones agropecuarias, la apuesta decidida por las producciones más rentables y cualificadas y su proyección manufacturera son piezas todavía estratégicas para el progreso agroindustrial. La provincia de Segovia está inmersa en un proceso de transformación de sus estructuras productivas que tiene que ver, como primera manifestación, con la actividad agraria y el desarrollo rural, y que encuentra en el entramado agroindustrial y en los productos agrarios de calidad su principal vector.

\section{BIBLIOGRAFÍA}

ALARIO, M. (2004). Diversificación económica en el medio rural: el turismo rural. En MOLINERO, F., MAJORAL, R., GARCÍA, J.M. y GARCÍA, G. (Coords.): Atlas de la España Rural. Madrid, Ministerio de Agricultura, Pesca y Alimentación, 355-363.

ALARIO, M. y BARAJA, E. (2006). Políticas públicas de desarrollo rural en Castilla y León, ¿sostenibilidad consciente o falta de opciones?: LEADER II. Boletín de la Asociación de Geógrafos Españoles, 41, 267-293.

ARFINI, F. (2006). Productos típicos y desarrollo rural: entre calidad y políticas de gobernanza. Estudios Agrosociales y Pesqueros, 210, 13-38.

ARMESTO, X.A. (2005). Notas teóricas en torno al concepto de postproductivismo agrario. Investigaciones Geográficas, 36, 137-156.

BARAJA, E., MOLINERO, F. y ALARIO, M. (2001). La aplicación de la Política Agraria Común en Castilla y León: Balance de una década. Valladolid, Departamento de Geografía de la Universidad de Valladolid.

BUTLER, R.W. (1998). Rural recreation and tourism. En ILBERY, B. (Ed.): The Geography of rural change. Harlow, Longman, 211-232.

CROUCH, D. (2006). Tourism, consumption and rurality. En CLOKE, P., MARSDEN, T. y MOONEY, P.H. (Eds.): Handbook of Rural Studies. London, Sage Publications, 355-364.

DELGADO, J.M ${ }^{\mathrm{a}}$. (2014). La creciente importancia de las actividades terciarias y la economía de servicios. En MARTíNEZ, L.C. y MORENO, A. (Eds.): La 
provincia de Segovia. Interpretación del espacio y definición del modelo territorial. Segovia, Diputación Provincial de Segovia, 155-242.

DELGADO, J.M ${ }^{\mathrm{a}}$. y MARTÍNEZ, L.C. (2014). Importancia de la población flotante en los municipios rurales del interior peninsular. Análisis de la situación en Castilla y León. Boletín de la Asociación de Geógrafos Españoles, 65, 207-229.

FERNÁNDEZ, J. (2011). Distribución y difusión espacio-temporal de la política de desarrollo rural en Castilla y León: de la Iniciativa LEADER I al LEADERCAL (1991-2013). Cuadernos de Geografía, 89, 49-70.

GARCÍA, F. (2010). Desarrollo y medio ambiente en un espacio en transformación: la provincia de Segovia. Madrid, Universidad Complutense de Madrid (Tesis Doctoral).

GALDOS, R. (2004). La intervención pública en la promoción de la calidad agroalimentaria: normativa comunitaria, española y vasca. Investigaciones Geográficas, 34, 47-62.

HORTELANO MÍNGUEZ, L.A. (2012). Las mudanzas y readaptaciones de la Sierra de Ayllón: la gestión sostenible del territorio y el fomento de las actividades turísticas. En DELGADO, C. y PLAZA, J.I. (Eds.): Territorio y paisaje en las montañas españolas. Estructuras y dinámicas espaciales. Santander, Librería Estudio y Ministerio de Ciencia e Innovación, 109-119.

ILBERY, B. y BOWLER, I. (1998). From agricultural productivism to postproductivism. En ILBERY, B. (Ed.): The Geography of rural change. Harlow, Longman, 57-84.

MARTIN, F. y MARTÍN, I. (2014). Los espacios rurales españoles: ¿territorios donde se produce y consume turismo o destinos sostenibles en entornos competitivos? El caso del Nordeste Segoviano. Boletín de la Asociación de Geógrafos Españoles, 64, 201-226.

MARTIN, Ma.A., LOZANO, M ${ }^{\mathrm{a}}$.J. y GONZÁLEZ, Mª.V. (2003). Los espacios protegidos como factor de localización del turismo rural en Castilla y León. Serie Geográfica, 11, 177-190.

MARTIINEZ, L.C. (2014). Un espacio provincial en proceso de transformación: modernización agraria y desarrollo rural. En MARTÍNEZ, L.C. y MORENO, A. (Eds.): La provincia de Segovia. Interpretación del espacio y definición del modelo territorial. Segovia, Diputación Provincial de Segovia, 37-89.

MAYA, A. (2008). El desarrollo rural como estrategia territorial: las perspectivas de los espacios rurales de Castilla y León. León, Secretariado de Publicaciones de la Universidad de León.

MAYA, A. e HIDALGO, C. (2009). Nuevas funciones y desarrollos de los territorios rurales europeos: su necesaria adaptación a métodos de producción duraderos $\mathrm{y}$ sostenibles. Boletín de la Asociación de Geógrafos Españoles, 49, 255-279.

MOLINA DE LA TORRE, I. y DELGADO URRECHO, J.M ${ }^{\mathrm{a}}$. (2014). Una estructura territorial contrastada. En MARTÍNEZ, L.C. y MORENO, A. (Eds.): La provincia de Segovia. Interpretación del espacio y definición del modelo territorial. Segovia, Diputación Provincial de Segovia, 353-414. 
MOLINERO, F. y ALARIO, M. (1994). La dimensión geográfica del desarrollo rural: una perspectiva histórica. Revista de Estudios Agrosociales, 169, 53-87.

MOLINERO, F., BARAJA, E. y RIVILLA, M. (Coords.) (2001). Inventario de productos agroalimentarios de calidad de Castilla y León. Valladolid, Junta de Castilla y León.

MOLINERO, F. (2011). Los paisajes del viñedo en Castilla y León: tradición, renovación y consolidación. Polígonos. Revista de Geografía, 21, 85-117.

MOLINERO, F. (2012). Las intensas transformaciones del mundo rural castellano y leonés: la marcha hacia el cuarto paradigma de desarrollo rural. En DELGADO, J.Ma. (Dir.): Población y poblamiento en Castilla y León. Valladolid, Consejo Económico y Social de Castilla y León, 557-636.

MOLINERO, F., HERRERO, D. y BARAJA, E. (2012). Significado y valor de los pagos "PAC" para el estudio de las estructuras agrarias en Castilla y León. Nimbus, 29-30, 431-446.

MOLTÓ, E. y HERNÁNDEZ, M. (2004). La funcionalidad de los medios rurales en las sociedades urbanas. Investigaciones Geográficas, 34, 63-76.

MUCHNIK, J. (2006). Identidad territorial y calidad de los alimentos: procesos de calificación y competencias de los consumidores. Agroalimentaria, 22, 89-98.

ORTEGA, J. (2004). La transición rural en España. El campo en la sociedad urbanizada. En NOGUÉS, S. (Ed.): El futuro de los espacios rurales. Santander, Servicio de Publicaciones de la Universidad de Cantabria, 90-114.

SANZ, G. (2006). Turismo en espacios protegidos: el Parque de las Hoces del Río Duratón (Segovia). En LACOSTA, A. (Ed.): Turismo y cambio territorial: ¿eclosión, aceleración, desbordamiento? Zaragoza, Universidad de Zaragoza, 291-302.

TOLÓN, A. y LASTRA, X. (2009). Los alimentos de calidad diferenciada. Una herramienta para el desarrollo rural sostenible. $M+A$. Revista electrónica de Medio Ambiente, 6, 45-67. 